\title{
SINGULAR VALUE INEQUALITIES FOR MATRICES WITH NUMERICAL RANGES IN A SECTOR
}

\author{
STEPHEN DRURY AND MINGHUA LIN
}

Abstract. Let $A=\left[\begin{array}{ll}A_{11} & A_{12} \\ A_{21} & A_{22}\end{array}\right]$, where $A_{22}$ is $q \times q$, be an $n \times n$ complex matrix such that the numerical range of $A$ is contained in $S_{\alpha}=\{z \in \mathbb{C}: \mathfrak{R} z>0,|\mathfrak{I} z| \leqslant(\mathfrak{R} z) \tan \alpha\}$ for some $\alpha \in$ $[0, \pi / 2)$. We obtain the following singular value inequality:

$$
\sigma_{j}\left(A / A_{11}\right) \leqslant \sec ^{2}(\alpha) \sigma_{j}\left(A_{22}\right), \quad j=1, \ldots, q,
$$

where $A / A_{11}:=A_{22}-A_{21} A_{11}^{-1} A_{12}$ and $\sigma_{j}(\cdot)$ means the $j$-th largest singular value. This strengthens some recent results on determinantal inequalities. We also prove

$$
\sigma_{j}(A) \leqslant \sec ^{2}(\alpha) \lambda_{j}(\Re A), \quad j=1, \ldots, n,
$$

where $\lambda_{j}(\cdot)$ denotes the $j$-th largest eigenvalue, complementing a result of Fan and Hoffman.

Mathematics subject classification (2010): 15A45.

Keywords and phrases: Singular value inequality, numerical range, accretive-dissipative matrix.

\section{REFERENCES}

[1] R. Bhatia, Matrix Analysis, GTM 169, Springer-Verlag, New York, 1997.

[2] R. Bhatia, F. Kittaneh, The singular values of $A+B$ and $A+i B$, Linear Algebra Appl. 431 (2009) $1502-1508$.

[3] S. W. DRURY, Fischer determinantal inequalities and Higham's Conjecture, Linear Algebra Appl. 439 (2013) 3129-3133.

[4] S. W. DRURY, M. Lin, Reversed Fischer determinantal inequalities, Linear Multilinear Algebra (2013). DOI: 10.1080/03081087.2013.804919

[5] R. A. Horn, C. R. Johnson, Matrix Analysis, Cambridge University Press, 1990.

[6] C.-K. LI, N. SZE, Determinantal and eigenvalue inequalities for matrices with numerical ranges in a sector, J. Math. Anal. Appl. 410 (2014) 487-491.

[7] M. Lin, Reversed determinantal inequalities for accretive-dissipative matrices, Math. Inequal. Appl. 12 (2012) 955-958.

[8] M. LIN, Fischer type determinantal inequalities for accretive-dissipative matrices, Linear Algebra Appl. 438 (2013) 2808-2812.

[9] M. Lin, D. ZHOU, Norm inequalities for accretive-dissipative operator matrices, J. Math. Anal. Appl. 407 (2013) 436-442. 